The AstrophysiCal Journal, 318:531-535, 1987 July 15

(C) 1987. The American Astronomical Society. All rights reserved. Printed in U.S.A.

\title{
DETECTION OF RETROGRADE GAS STREAMING IN THE SB0 GALAXY NGC $4546^{1}$
}

\author{
G. Galletta \\ Astronomy Department, University of Padua \\ Received 1986 November 12; accepted 1986 December 18
}

\begin{abstract}
We report spectroscopic observations of the almost edge-on SB0 galaxy NGC 4546 which reveal a striking discordance between the derived emission and absorption-line velocities. The gas clouds show velocities that are similar in amplitude but opposite in direction from the stars. This discordance is seen in observations obtained through slits oriented in a wide range of position angles. NGC 4546 is thus, at present, unique as a disk system exhibiting large-scale retrograde motions relative to the stellar component.

Orbits elongated both along the bar major axis (prograde, stars) and along the bar intermediate axis (retrograde, gas) are found. The possibility that this material originated from an infall is discussed.

Subject headings: galaxies: internal motions — galaxies: individual (NGC 4546)
\end{abstract}

\section{INTRODUCTION}

The presence of ionized gas and dust in S0 galaxies is not rare: optical emission lines are detected in $\sim 48 \%$ of field SO galaxies (Gisler 1978), while dust lanes are a key feature in their morphological classification (Sandage 1961, p. 10; de Vaucouleurs 1959). But in comparison with the spirals, their disks are quite gas poor, suggesting the presence of a mechanism for gas depletion whose nature is still under debate (see White 1982). We present here observations of a barred S0, namely NGC 4546, where there is some evidence for external gas enrichment. It was selected for kinematical study because of the following properties: (i) it is bright; $m_{B} \approx 11.3$; (ii) its ellipticity, which at the $25 \mathrm{mag} \operatorname{arcsec}^{-2}$ isophote is 0.52 , indicates that it is almost edge-on, enabling us to study the bar along one of its principal planes; (iii) it shows the presence of [O II] lines at $\lambda \lambda 3727-29$ (Humason, Mayall, and Sandage 1956), and (iv) it possesses an $\mathrm{H}$ I rotating disk with a velocity width for the line of $350 \mathrm{~km}$ $\mathrm{s}^{-1}$ (Bieging 1978). In addition, it is a member of a small and relatively close group (Virgo V), whose distance is estimated to be $\sim 17.8 \mathrm{Mpc}$ (de Vaucouleurs 1975).

We report the first results of our analysis of NGC 4546 where we discovered a striking discordance between the derived emission and absorption-line velocities, indicating large-scale gaseous and stellar motions similar in amplitude but opposite in direction. Near the center, stellar and gas orbits appear elongated in the direction of the major and intermediate axis of the bar, respectively.

In the literature, many elliptical galaxies possessing gas, or dust lanes like NGC 5128, show rotation axes of gas and stars mutually perpendicular (see Bertola [1985] for a review). The only disk galaxies showing gas in orbits independent from that of the stars are the S0 galaxies with polar rings discussed by Schweizer, Whitmore, and Rubin (1983), whose prototype is NGC 2685. In these galaxies a luminous disk of gas is circling a stellar body with S0 morphology, again with a rotation axis almost perpendicular to that of the stars. But in only two cases, the E0 NGC 5898 (Bettoni 1984) and the E4 NGC 7097 (Caldwell, Kirshner, and Richstone 1986) does the gas motion appear to be retrograde with respect to the slow rotation of the stars. NGC 4546 is thus, at present, unique as a disk system

\footnotetext{
${ }^{1}$ Based on observations collected at the European Southern Observatory, La Silla, Chile.
}

exhibiting large-scale retrograde motions relative to the stellar component.

\section{OBSERVATIONS AND DATA ANALYSIS}

NGC 4546 has been studied from 1986 May 4-6 with the Boller and Chivens spectrograph on the $2.2 \mathrm{~m}$ ESO-MPI telescope. Observations at five different position angles were recorded on a $320 \times 512$ pixel CCD. The exposure times range from 90 minutes along the minor axis (P.A. $=168^{\circ}$ ) and intermediate position angles $\left(\right.$ P.A. $=33^{\circ}, 98^{\circ}$, and $135^{\circ}$ ) to 120 minutes for the major axis $\left(\right.$ P.A. $\left.=78^{\circ}\right)$. The spectral resolution is $1.77 \AA$ pixel $^{-1}$, while the scale perpendicular to the dispersion is $1^{\prime \prime} 8$ pixel $^{-1}$ or $59^{\prime \prime} \mathrm{mm}^{-1}$. The wavelength interval studied is $4900-5700 \AA$. In order to study the distribution of the gas as well as its velocity structure outside of the principal galactic plane two spectra were also obtained offset from the nucleus of the galaxy by $5^{\prime \prime}$ (60 minute exposures NW and SE). An additional major-axis spectrum of 15 minute exposure was kindly obtained for us with the same instrument by $\mathrm{S}$. di Serego on May 6/7 at lower dispersion $\left(5.16 \AA\right.$ pixel $\left.^{-1}\right)$ in the interval 4730-7300 $\AA$.

In addition to the spectroscopic data, some $V$-frames (exposures of 1 and 15 minutes) were recorded with the $320 \times 512$ RCA CCD of the $1.5 \mathrm{~m}$ Danish telescope on the nights of 1986 May 6/7 and 7/8, to study the morphology of the galaxy. The 1 minute image is reproduced in Figure 1, along with the slit orientations corresponding to the spectra obtained. Reproductions of the major- and minor-axis spectra are also shown in Figure 1. Some additional images of this galaxy and of the spectra have been published elsewhere (Galletta 1986).

In our higher dispersion spectra the [O III] $\lambda \lambda 4959-5007$ emission lines are visible, but $\lambda 4959$ is too weak to yield useful velocity measures. It is also confused by many galaxy absorption features which intersect it with opposite inclination (see Fig. 1). The absorption features $\mathrm{Mg}$ I $\lambda 5175.4, \mathrm{Ca}+\mathrm{Fe} \lambda 5269.0$, and $\mathrm{Fe} \lambda 5331.5$ are the strongest absorption lines in the spectra and have been used in the velocity determination of the stars. In the low-dispersion spectrum $\mathrm{H} \alpha,[\mathrm{N}$ II $] \lambda \lambda 6548-84$ and [S II] $\lambda \lambda 6717-31$ emission are also visible. While less accurate, the galaxy rotation measures obtained from these lines are in agreement with those coming from the major axis spectrum at higher resolution. 

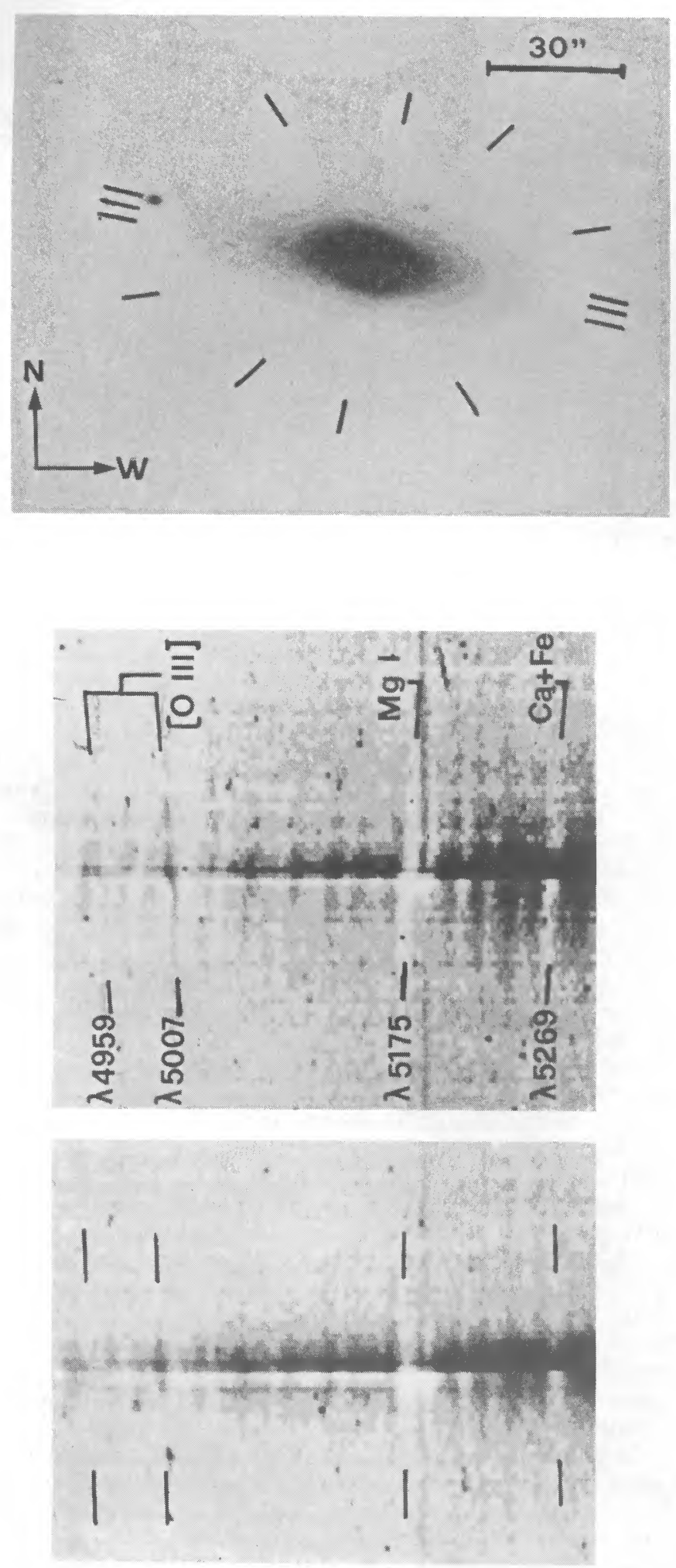

FiG. 1.-Top: One-minute CCD exposure of NGC 4546 (1.52 m Danish ESO telescope, $V$-band) showing the positions of the spectrograph slits relative to the galaxy body. Middle: reproductions of the major-axis spectrum $(120 \mathrm{~m}$, $2.2 \mathrm{~m}$ ESO-MPI telescope). Note that the gas lines appear inclined in a direction opposite to that of the stellar lines, suggesting opposite direction of motion. Bottom: Minor-axis spectrum. All images have the same scale, and the continuum has been partially subtracted to make the lines more evident. The $4959 \AA$ line is faint and only slightly visible.
All the data presented here comes from a reduction made at the Padova HP computer center using the ESO IHAP package. The velocities measured were obtained by fitting Gaussian profiles to the emission and absorption lines of each spectrum for each scan line. The resulting velocities both for the gaseous and the stellar component of the galaxy are mapped in the two panels of Figure 2, together with the inner isophotes of the galaxy image, plotted at the same scale. The most part of the velocity values have an rms error $\sigma_{v} \leq 25 \mathrm{~km}$ $\mathrm{s}^{-1}$, with some point having $\sigma_{v} \leq 50 \mathrm{~km} \mathrm{~s}^{-1}$ and very few points with higher uncertainty. In Figure 2, all values are scaled to the systemic velocity deduced from our spectra. The estimates made independently for the gas and for the stars gave a systemic velocity equal to $1021 \pm 18$ and $1013 \pm 26$, respectively. Lines of equal velocity have been drawn in Figure 2, interpolating by eye from the observed velocities (with slight visual smoothing based upon the local uncertainty) in order to estimate the galaxy velocity fields. the following kinematical discussion is based mainly on these maps.

\section{DISCUSSION}

In most of the spectra, the gas lines appear inclined in a direction opposite to that of the stellar lines, suggesting opposite direction of motion (see Fig. 1). This fact characterizes the uniqueness of the kinematics in this stellar system. Small gas/stars velocity discordances $\left(\Delta v \approx 50-100 \mathrm{~km} \mathrm{~s}^{-1}\right)$ have been seen in the nuclear regions of some spiral galaxies (see, e.g., Sulentic and Arp 1987). At the present time, no other S0 or spiral galaxy for which both the gas and the star kinematics have been investigated is known to possess this widespread characteristic. The behavior is seen in most of the spectra taken at different position angles, indicating that the phenomenon is quite extended.

Concerning the optical morphology shown in Figure 1, at the opposite ends of the major axis two bright extensions are visible, similar to the spindles of the edge-on disk galaxies. A faint dust lane is observable along the major axis at $7^{\prime \prime}-10^{\prime \prime}$ from the nucleus. The bar, whose presence was noted by de Vaucouleurs, de Vaucouleurs, and Corwin (1976) and Sandage and Tammann (1981), appears just like a broad and hazy enhancement of the central surface brightness, slightly elongated at $45^{\circ} \mathrm{NE}$ of the major axis $\left(\right.$ P.A. $=33^{\circ}$ ). In the longer exposure it is completely embedded in the galaxy body, whose isophotes are rounder with an axial ratio of $\sim 0.43$. This value, compared with the mean intrinsic flattening of disk galaxies (0.25 according to Sandage, Freeman, and Stokes 1970), and the presence of a spindle allows us to assume that the plane of the galactic disk is seen nearly edge-on. Its inclination is, thus, probably greater than $60^{\circ}$ with respect to the plane of the sky. This value is in agreement with the observed deviation of the bar from the apparent major axis, under the usual assumption that the bar is elongated in the disk equatorial plane. In the following discussion, we shall assume an upper limit to the inclination of the galactic plane with respect to the sky of $\sim 60^{\circ}$. The measured velocities then cannot differ from the true velocities on the plane of the galaxy by more than a factor of $15 \%$.

\section{a) The Stellar Velocity Field}

A look at the Figure 2 shows that in the velocity field of the stars the NE side of the galaxy is receding while the SW side is approaching. Some deviations from circular motions are visible: (i) near the center, the isovelocity lines with $|v|<150$ 

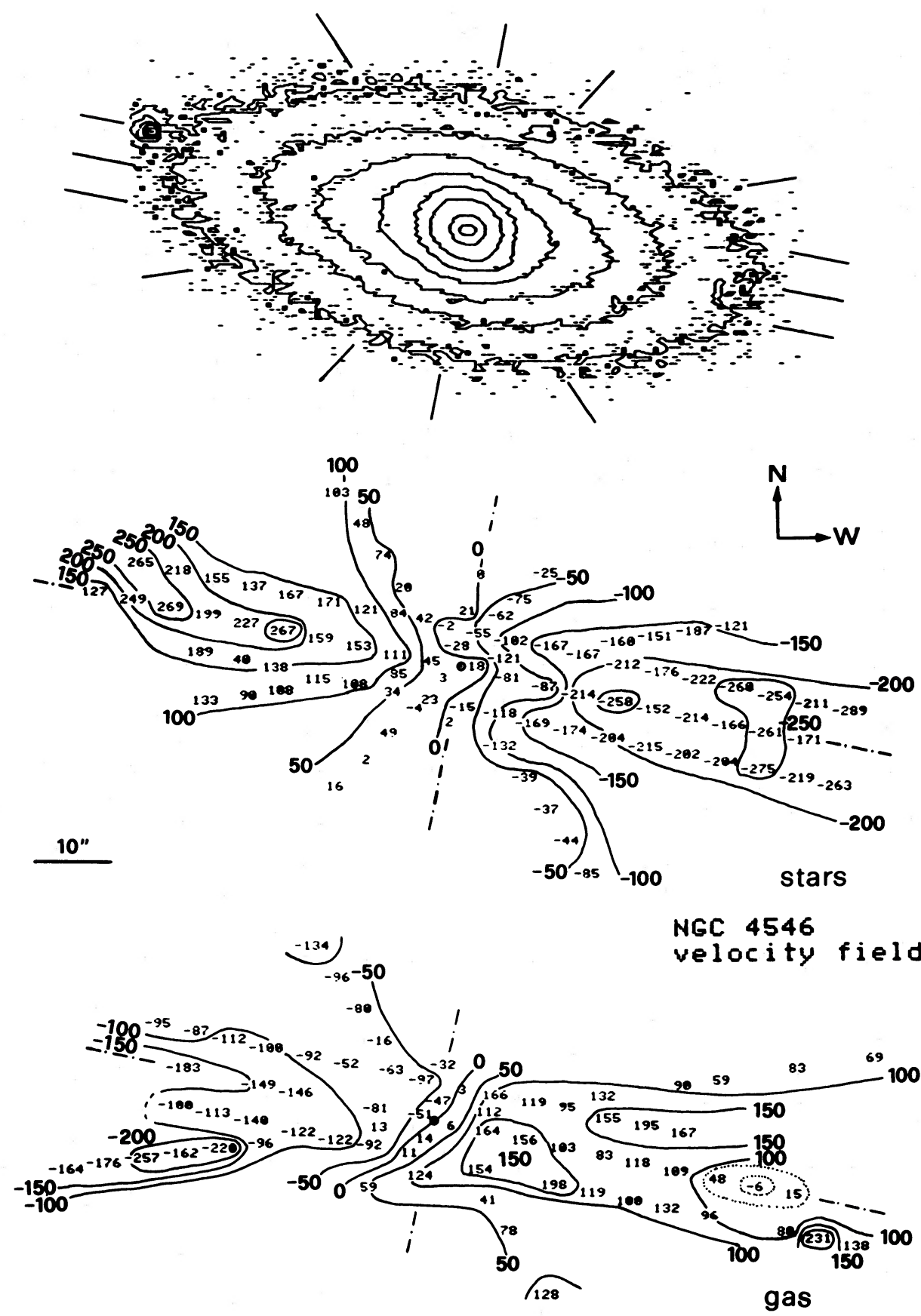

Fig. 2.-Top panel: isophotes of the central part of NGC 4546, with the positions of the spectrograph slit drawn at the boundary. Middle and bottom panels: measured velocities for stars and gas, respectively, rebinned in intervals of 5" each and referred to the systemic velocity $\left(\sim 1017 \mathrm{~km} \mathrm{~s}^{-1}\right)$. All panels have the same scale and orientation. Hand fits of the lines of equal velocity are also shown.

$\mathrm{km} \mathrm{s}^{-1}$ and within $\sim 15^{\prime \prime}$ from the nucleus appear to assume an orientation parallel to the bar major axis. Because of the small velocities involved, this feature should be in doubt for isovelocity lines NE of the nucleus, as well as for the zero velocity line. Comparison of the isophotes reveals that this is exactly the region were the presence of the bar distorts the isophotes; (ii) along the bar, an excess of velocity in the same direction as the general rotation is present at larger radii, giving again straight velocity contours. The elongation of the isovelocity lines along the bar is a feature already observed in other barred galaxies (Peterson et al. 1978; Kormendy 1983). It is also in agreement with the expectation of barred galaxy models for elliptical patterns of flow (Huntley 1978; Miller and Smith 1979).

Outside of the central regions, more regular motions predominate. The line of zero velocity coincides with the apparent minor axis of the galaxy, and the stellar velocity gradient increases in agreement with the expectation for orbits lying in the plane of the galaxy. It reaches a maximum of $\sim 12 \mathrm{~km} \mathrm{~s}^{-1}$ $\operatorname{arcsec}^{-1}$ at P.A. $=80^{\circ}$, very close to the major axis of the galaxy. The major axis rotation curve reaches a first maximum at $22^{\prime \prime}$ from the center in both sides, with a velocity of $\sim 260 \mathrm{~km}$ 
$\mathrm{s}^{-1}$ and a second maximum of $\sim 270 \mathrm{~km} \mathrm{~s}^{-1}$ at $40^{\prime \prime}$ from the center.

\section{b) The Gaseous Velocity Field}

The gas clouds in NGC 4546 appear on the contrary to move in a velocity field completely decoupled from that of the stars (Fig. 2). Neither the direction nor the orientation of the zero velocity lines are similar in the two cases. The NE side of the galaxy is approaching, while the SW is receding. In addition, this velocity field shows a more complicated structure: (i) the zero velocity line and all the isovelocity contours within $6^{\prime \prime}$ of the center appear aligned along P.A. $=123^{\circ}$, which coincides with the apparent minor axis of the bar; (ii) despite this fact, the line of maximum gradient is not reached along the bar major axis but closer to the major axis, at P.A. $=225^{\circ}$. In this direction the (asymmetric) velocity trend reaches $\sim 200 \mathrm{~km} \mathrm{~s}^{-1}$ at 17 " SW of the nucleus; (iii) peaks and dips of rotation appear in many places along the galaxy, indicating a superposition of streaming motions with different value and orientations. For instance, a marked dip is present at $42^{\prime \prime} \mathrm{SW}$ of the center, where the velocities fall from $100 \mathrm{~km} \mathrm{~s}^{-1}$ down to the systemic velocity.

Examination of the two-dimensional spectral images (Galletta 1986) shows that the gas is nonuniformly distributed. It appears confined within an asymmetric structure (disk?) of projected dimension $1.7 \times 8.2 \mathrm{kpc}$, not aligned with the stellar disk of the galaxy or with the bar.

The features (i) and (ii) are both indications of elliptic flows similar to that observed for the stars. But since in this case the orientation of the orbits is parallel to the apparent minor axis of the bar we must deduce that these orbits extend along the intrinsic intermediate axis of the bar. Actually, this configuration is predicted for some families of retrograde orbits in tumbling potentials, either in the case where they lie on the equatorial plane (Contopoulos and Papayannopoulos 1980; de Zeeuw and Merrit 1983) or where they are within planes that are warped in the outer parts (anomalous orbits; van Albada, Kotanyi, and Schwarzschild 1982; Merrit and de Zeeuw 1983). We note that a warping of the gas plane, with innermost orbits on the equatorial plane of the disk and outermost orbits in the direction of the velocity maxima observed NW and SE of the nucleus, could explain some of the asymmetries presented in the point (iii), suggesting chaotic or noncoplanar motions of the gas clouds in the outer parts of the studied region of the galaxy.

\section{c) Modeling NGC 4546}

As an S0, NGC 4546 is very peculiar: the gas possesses motions independent from those of the stars, opposite in direction and therefore probably not confined to the principal plane. The retrograde circulation is a large-scale phenomenon, involving at least $8 \mathrm{kpc}$ of the galaxy. In the inner regions, the bar potential has probably modified the motions of stars and gas, confining them to the corresponding families of orbits which lie on or near the plane of the galaxy. Here the stars move along the bar major axis, while the (retrograde) gas orbits are elongated toward the intermediate axis of the bar. Considering these differences, we find it difficult to conceive of a mechanism that in a collapsing protogalaxy will discriminate between newly born stars and the residual gas, driving them in two opposite directions of rotation. The same is true if we postulate this gas to be the result of stellar mass loss. For these reasons we favor an interpretation where the gas in the stellar body of NGC 4546 was acquired externally. Further that the acquisition of gas occurred when the galaxy was already an S0.

As discussed in the Introduction, rotation axes of gas and stars mutually perpendicular have been observed both in elliptical galaxies with dust (see Bertola 1985) and in the S0 galaxies with polar rings (Schweizer, Whitmore, and Rubin 1983). In addition, two cases of elliptical galaxies are known where these axes are even antiparallel. The origin of the peculiar kinematics for all these galaxies-E or S0's-has been ascribed to a past collision with a smaller companion (Bertola 1985; Schweizer et al. 1983).

If we consider the kinematical similarity between the above systems and NGC 4546, we could make the hypothesis that it has undergone a past collision with a gas-cloud or a gas-rich system. To produce the observed motions, the orbital angular momentum of this small system and (eventually) its spin should be opposite to the spin of the stellar orbits in NGC 4546. In this scheme, it is possible to estimate that, for a small impact angle, the gas would have had time to settle toward the main plane in the central part of the galaxy, where the galactic potential is stronger, because of the differential precession of its orbits induced by the disk. This is a reasonable assumption, since the central orbits appear quite ordered in the corresponding elongated patterns and the nonregular inclined orbits are confined to the outer parts. With the typical gas velocities observed in the galaxy we estimate that at $\sim 2.5 \mathrm{kpc}$ from the center the rotational period is of the order of 40-50 million years. Neglecting the presence of the bar, it is possible to estimate for an oblate potential that the settling time into the equatorial plane is only a few hundred million years (SteimanCameron and Durisen 1987), very short compared to the lifetime of a galaxy. In the above computation we assumed an impact angle for the falling gas of $40^{\circ}$ with respect to the plane of the disk. Highly inclined collisional orbits would, of course, generate longer settling times. If some stars were present in the colliding galaxy, they would disperse in the main body of the surviving galaxy (Toomre 1977; Schweizer et al. 1983). Perhaps the lower stellar velocities observed SE of the major axis, where the gas motions show a local maximum in opposite direction, could be ascribed to these stellar debris in retrograde motion. In any case, only in an E or S0, which is relatively free from the presence of dense gas clouds, could the gas orbits survive without being destroyed because of the collision with the preexistent clouds. No X-ray observations of this galaxy are known in the literature. If this is due to a lack of X-ray emission, it suggests that NGC 4546 was extremely gas poor before the proposed event.

An alternative to this capture hypothesis is that this material cames from a tidal encounter with a close system, still surviving after the collision. Together with NGC 4691 (type S0/a), NGC 4546 is the only galaxy in the Virgo $\mathrm{V}$ cloud to possess an S0 morphology, the other members being late-type spirals (de Vaucouleurs 1975). Since the members of the group at the assumed distance of $17.8 \mathrm{Mpc}$ have a projected separation from NGC 4546 of $\sim 0.9-1.2 \mathrm{Mpc}$, it is unlikely that this material comes from one of them. In fact, assuming relative velocities as great as $400 \mathrm{~km} \mathrm{~s}^{-1}$, the epoch of a closest approach cannot be lower than $2.5 \mathrm{Gyr}$, one order of magnitude greater than the expected settling time. In addition, none of these systems, especially the spiral ones, show perturbations of the gas morphology that can justify this hypothesis. Another possibility is the collision with a dwarf satellite that could be lost among the several faint galaxies, probably background 
objects, present around NGC 4546. The closest one, ZWG 014.074 (Zwicky, Herzog, and Wild 1961) is an elliptical-like system of magnitude 15.7 with a bright center and a diffuse body of extension $9^{\prime \prime} \times 21^{\prime \prime}$. If it is at the same distance of NGC 4546 , the projected separation between the two systems is $\sim 29$ $\mathrm{kpc}$, short enough to justify a past interaction. On the basis of a contrast enhanced picture of the field produced at the ESO Photo Lab from the glass copy of Palomar Sky Survey, there is an indication of a faint bridge of matter connecting this galaxy with NGC 4546, but the quality of the material does not allow any definitive conclusion on the closeness between the two systems. Searches for newly formed stars in the UV and for H I bridges are needed to better understand the nature of the phenomena observed in this peculiar galaxy.

Many thanks are due to S. di Serego for providing us the low dispersion spectrum of NGC 4546. It is a pleasure to thank also F. Bertola and J. Sulentic for helpful discussions on this paper, A. Satta of the Padova photo lab for the preparation of the figures, and C. Madsen of the ESO photo lab for the contrast enhanced print of the field.

\section{REFERENCES}

Bertola, F. 1985, in IAU Symposium 127, Structure and Dynamics of Elliptical Galaxies, ed. T. de Zeeuw (Dordrecht: Reidel), p. 1.

Bettoni, D. 1984, Messenger, 37, 17.

Bieging, J. H. 1978, Astr. Ap., 64, 26.

Caldwell, N., Kirshner, R. P., and Richstone, D. 1986, Ap. J., 305, 136.

Contopoulos, G., and Papayannopoulos, Th. 1980, Astr. Ap., 92, 33

de Vaucouleurs, G. 1959, in Handbuch der Physik, 53, ed. S. Flügge (New York: Springer), p. 278.

A. 1975, in Stars and Stellar System Vol. 9, Galaxies and the Universe, ed. A. Sandage, M. Sandage, and J. Kristian (Chicago: University of Chicago Press), p. 584.

de Vaucouleurs, G., de Vaucouleurs, A., and Corwin, H. G., Jr. 1976, Second

Reference Catalog of Bright Galaxies (Austin: University of Texas Press) (RC2).

de Zeeuw, T., and Merrit, D. 1983, Ap. J., 267, 571.

Galletta, G. 1986, The Messenger, 45, 18

Gisler, G. R. 1978, M.N.R.A.S., 183, 633 .

Humason, M. L., Mayall, N. V., and Sandage, A. R. 1956, Astr. J., 61, 101.

Huntley, J. M. 1978, Ap. J. (Letters), 225, L101.

Kormendy, J. 1983, Ap. J., 275, 529.

Merrit, D., and de Zeeuw, T. 1983, Ap. J., 267, L19.
Miller, R. H., and Smith, B. F. 1979, Ap. J., 227, 785.

Peterson, C. J., Rubin, V. C., Ford, W. K., and Thonnard, N. 1978, Ap. J., 219, 31.

Sandage, A. 1961, The Hubble Atlas of Galaxies (Washington, DC: Carnegie Institute of Washington).

Sandage, A., Freeman, K. C., and Stokes, N. R. 1970, Ap. J., 160, 831.

Sandage, A., and Tammann, G. A. 1981, A Revised Shapley-Ames Catalog of Bright Galaxies, (Washington, DC: Carnegie Institute of Washington).

Schweizer, F., Whitmore, B. C., and Rubin, V. C. 1983, A.J., 88, 909.

Steiman-Cameron, T. Y., and Durisen, R. H. 1987, Ap. J., submitted.

Sulentic, J., and Arp, H. 1987, Ap. J., submitted.

Toomre, A. 1977, in The Evolution of Galaxies and Stellar Populations, ed. R. B. Larson and B. M. Tinsley (New Haven: Yale University Press).

van Albada, T. S., Kotanyi, C. G., and Schwarzschild, M. 1982, M.N.R.A.S., 198, 303.

White, S. D. M. 1982, in Saas-Fee 12th Adv. Course, Morphology and Dynamics of Galaxies, ed. L. Martinet and M. Mayor (Geneva: University of Geneva), p. 379.

Zwicky, F., Herzog, E., and Wild, P. 1961, Catalogue of Galaxies and Cluster of Galaxies Vol. 1 (Pasadena: California Institute of Technology), p. 50.

G. Galletta: Dipartimento di Astronomia dell'Università di Padova, Vicolo dell'Osservatorio 5, 35122 Padova, Italy 